\title{
Oscillation Criteria for Solutions of Neutral Differential Equations of Impulses Effect with Positive and Negative Coefficients
}

Hussain Ali Mohamad

Received 22/2/2019, Accepted 24/11/2019, Published 1/6/2020

Aqeel Falih Jaddoa ${ }^{2 *}$ c) (7)

This work is licensed under a Creative Commons Attribution 4.0 International License.

\begin{abstract}
:
In this paper, some necessary and sufficient conditions are obtained to ensure the oscillatory of all solutions of the first order impulsive neutral differential equations. Also, some results in the references have been improved and generalized. New lemmas are established to demonstrate the oscillation property. Special impulsive conditions associated with neutral differential equation are submitted. Some examples are given to illustrate the obtained results.
\end{abstract}

Key words: Impulsive Neutral Differential Equations, Oscillation, Variable Delays, Variable Coefficients.

\section{Introduction}

The oscillatory theory of impulsive delay differential equations is appearing as an important field of investigation, because it is much richer than the theory of delay differential equations without impulses effects. The differential equations with impulses effects describe the process of evolution that rapidly changes their state at certain moments; therefore this type of differential equations is suitable for the mathematical simulation of the evolutionary process in which the parameters are subject to relatively long periods of smooth variation followed by rapid short-term change.

The wide possibility of applications determines the increasing interest in impulsive differential equations. The importance of the need to study differential equations with impulsive is due to the fact that these equations are more comprehensive in their use of mathematical modeling where gaps in the model can be addressed by limiting these gaps in specific points called the points of impulses (which are not continuous points) in many real processes and phenomena studied in control theory, biology, mechanics, medicine, electronic, economic, etc.

For instance, there are a lot of applications of impulsive differential equations in neural networks (1-4), n control theory (5), in biology (6) and economics (7).

\footnotetext{
${ }^{1}$ Department of Mathematics, College of Science for Women, University of Baghdad.

${ }^{2}$ Department of Mathematics College of Education for Pure Science Ibn Al-Haitham, University of Baghdad. *Corresponding author: aqeel.f.j@ihcoedu.uobaghdad.edu.iq *ORCID ID: https://orcid.org/0000-0002-5590-7991
}

Mohamad et al. (8) obtained sufficient conditions for the oscillation of all solutions of neutral differential equations with variable delays, while $\mathrm{H}$. Chen et al (9) studied the existence of solutions for impulsive differential equations. Isaac (10) classifies non-oscillatory solutions of impulsive differential equations of the second order. So many papers focused on studying the oscillation of impulsive neutral differential equations and impulsive systems $(11,12)$.

The main purpose of this paper is to study one of the important properties of the solution for the impulsive neutral differential equations with positive and negative coefficients, it is the oscillation property. The sufficient conditions to guarantee the oscillation of all solutions for the impulsive neutral differential equation with positive and negative coefficients have been obtained.

Consider the impulsive neutral differential equation of form:

$$
\left.\begin{array}{c}
{[x(t)-P(t) x(\tau(t))]^{\prime}+Q(t) x(\sigma(t))} \\
-R(t) x(\alpha(t))=0 \\
t \neq t_{k} \quad k=1,2, \ldots \\
x\left(t_{k}^{+}\right)+b_{k} x\left(t_{k}\right)=a_{k} x\left(t_{k}\right), \\
t=t_{k}, \quad k=1,2, \ldots
\end{array}\right\}
$$

Where $t_{k}$ are the moments of impulses effect. The numbers $a_{k}$ and $b_{k}$ are positive real numbers,

$k=1,2, \ldots, P \in P C\left(\left[t_{0}, \infty\right), R^{+}\right), \quad$ where $P C\left(\left[t_{0}, \infty\right), R^{+}\right)$denotes the set of all functions $f:\left[t_{0}, \infty\right) \rightarrow R^{+}$such that $f$ is continuous for $t \neq t_{k}, \quad k=1,2, \ldots$ and $f\left(t_{k}^{-}\right)=\lim _{t \rightarrow t_{k}^{-}} f(t)$.

Let $Q, R \in C\left(\left[t_{0}, \infty\right), R^{+}\right), \quad$ and $\tau, \sigma, \alpha$ are continuous strictly increasing functions with $\lim _{t \rightarrow \infty} \tau(t)=\infty, \lim _{t \rightarrow \infty} \alpha(t)=$ 
$\infty, \lim _{t \rightarrow \infty} \sigma(t)=\infty . \quad$ The functions $\tau^{-1}(t), \sigma^{-1}(t), \alpha^{-1}(t)$ are the inverse of the functions $\tau(t), \alpha(t), \sigma(t)$ respectively, $\tau(t)<t$. The initial function has defined: $x(t)=\omega(t), t \in\left[\rho\left(t_{0}\right), t_{0}\right]$, $\rho(t)=\min _{t \geq t_{0}}\{\tau(t), \alpha(t), \sigma(t)\}$, where $\omega(t) \in P C\left(\rho\left(t_{0}\right), R\right)$.

Definition 1: A function $f$ is piecewise continuous on $[a, b]$ if

(a) $f\left(x_{0}{ }^{+}\right)$exists for all $x_{0}$ in $[a, b)$;

(b) $f\left(x_{0}^{-}\right)$exists for all $x_{0}$ in $(a, b]$;

(c) $f\left(x_{0}{ }^{+}\right)=f\left(x_{0}{ }^{-}\right)=f\left(x_{0}\right)$ for all but finitely many points $x_{0}$ in $(a, b)$;

If (c) fails to hold at some $x_{0}$ in $(a, b), f$ has a jump discontinuity at $x_{0}$. Also, $f$ has a

jump discontinuity at $a$ if $f\left(a^{+}\right) \neq f(a)$ or at $b$ if $f\left(b^{+}\right) \neq f(b)$.

Definition 2: A function $x(t)$ is said to be a solution of Eq.(1) which is satisfying the initial condition if

$A 1: x(t)=\omega(t)$ for $\rho\left(t_{0}\right) \leq t \leq t_{0}$ and $x(t)$ is continuous for $t \geq t_{0}$ and $t \neq t_{k}$,

$k=1,2, \ldots$.

A2: $x(t)-P(t) x(\tau(t))$ is piecewise continuously differentiable $t \geq t_{0}$ and

$$
t \neq t_{k}, \quad t \neq \tau^{-1}\left(t_{k}\right), \quad t \neq \sigma^{-1}\left(t_{k}\right),
$$

$t \neq \alpha^{-1}\left(t_{k}\right) k=1,2, \ldots$, and satisfies Eq.(1);

A3: $x\left(t_{k}^{+}\right)$and $x\left(t_{k}^{-}\right)$exist with

$x\left(t_{k}^{-}\right)=x\left(t_{k}\right)$ and satisfies impulsive Eq.(1) where

$$
x\left(t_{k}^{+}\right)=\lim _{t \rightarrow t_{k}^{+}} x\left(t_{k}\right) \quad \text { and } \quad x\left(t_{k}^{-}\right)=
$$

$\lim _{t \rightarrow t_{k}^{-}} x\left(t_{k}\right)$.

Definition 3: The function $f(t):\left[t_{0}, \infty\right) \rightarrow R$ is said to be eventually enjoy the property $P$ if there exist an interval $\left[\bar{t}_{0}, \infty\right) \subset\left[t_{0}, \infty\right)$ in which $f(t)$ enjoys the property $P$ for $t \geq \overline{t_{0}}$. So a function $x(t) \in \mathrm{C}\left(\left[t_{0}, \infty\right), R\right)$ is said to be eventually positive (negative), if there exist $t_{1} \geq t_{0}$ such that $x(t)>0(x(t)<0)$ for all $t \geq t_{1}$.

Definition 4: A regular solution $x(t)$ of eq. (1) is said to be oscillatory in $\left[t_{0}, \infty\right)$ if it has arbitrarily large zeros for $t \geq t_{1} \geq t_{0}$, that is, there exist a sequence of zeros $\left\{t_{n}\right\}$ such that $\lim _{n \rightarrow \infty} t_{n}=\infty$ where $x\left(t_{n}\right)=0$, otherwise $x(t)$ is said to be nonoscillatory on $\left[t_{1}, \infty\right)$, that is $x(t) \neq 0$ for each $t \geq t_{1}$, which means that either $x(t)$ is eventually positive, or is eventually negative.

\section{Some Basic Lemmas:}

The following lemmas will be useful to prove the main results:

Lemma 1: (13) Suppose that $g, h:\left[t_{0}, \infty\right) \rightarrow R$ are continuous functions, $g(t) \geq 0$ eventually, $h(t) \geq$ $t$ and $h^{\prime}(t) \geq 0$ for $t \geq t_{0}$. If

$$
\liminf _{t \rightarrow \infty} \int_{t}^{h(t)} g(s) d s>\frac{1}{e}
$$

then the inequality $x^{\prime}(t)-g(t) x(h(t)) \geq 0$ has no eventually positive solutions.

Lemma 2: (13) Suppose that $g, h \in\left[t_{0}, \infty\right) \rightarrow$ $[0, \infty), h(t) \leq t$ and $\lim _{t \rightarrow \infty} h(t)=\infty$. If

$$
\liminf _{t \rightarrow \infty} \int_{h(t)}^{t} g(s) d s>\frac{1}{e}
$$

then the inequality

$x^{\prime}(t)+g(t) x(h(t)) \leq 0$

has no eventually positive solutions.

The following lemma is a generalization of the lemma 1.5.4 (14).

Lemma 3: Assume that

I. $f, g, y, \tau, \gamma \in C\left[\left[t_{0}, \infty\right), R\right], f(t)<0$, $\lim _{t \rightarrow \infty} f(t)$ exist, $0<g(t) \leq 1$,

$\tau(t)<t, \gamma(t) \geq t, t \geq t_{0}, \lim _{t \rightarrow \infty} \tau(t)=\infty$ and

$$
\left.\begin{array}{c}
y(t) \leq f(t)+ \\
g(t) \max \{y(s): \tau(t) \leq s \leq \gamma(t)\}, \\
t \geq t_{0}
\end{array}\right\}
$$

Then $y(t)$ cannot be positive for $t \geq t_{1} \geq t_{0}$.

II. $f, g, y, \tau, \gamma \in C\left[\left[t_{0}, \infty\right) ; R\right], f(t)>0$, $\lim _{t \rightarrow \infty} f(t)$ exist, $0<g(t) \leq 1, \tau(t)<t$, $\gamma(t) \geq t, t \geq t_{0}, \lim _{t \rightarrow \infty} \tau(t)=\infty$ and

$$
\left.\begin{array}{c}
y(t) \geq f(t)+ \\
g(t) \min \{y(s): \tau(t) \leq s \leq \gamma(t)\}, \\
t \geq t_{0}
\end{array}\right\}
$$

Then $y(t)$ cannot be negative for $t \geq t_{1} \geq t_{0}$

Proof. The part (I) has been proved, proof of (II) is similar and will be omitted. For the sake of contradiction assume that $y(t)>0, t \geq t_{0}$, it follows that $y(t)$ must be bounded, otherwise, there exists a sequence $\left\{t_{n}\right\}$,

$\lim _{n \rightarrow \infty} t_{n}=\infty, \lim _{n \rightarrow \infty} y\left(t_{n}\right)=\infty$ and

$$
y\left(t_{n}\right)=\max \left\{y(s): t_{0} \leq s \leq \gamma\left(t_{n}\right)\right\}
$$

Which is possible since $y\left(t_{n}\right) \rightarrow \infty$, and there exists $n_{1}$ such that $\tau\left(t_{n}\right) \geq t_{0}$ for $n \geq n_{1}$ the inequality (4) reduces:

$$
\begin{gathered}
y\left(t_{n}\right) \leq \\
f\left(t_{n}\right)+g\left(t_{n}\right) \max \left\{y(s): \tau\left(t_{n}\right) \leq s \leq \gamma\left(t_{n}\right)\right\} \\
\leq f\left(t_{n}\right)+g\left(t_{n}\right) \max \left\{y(s): t_{0} \leq s \leq \gamma\left(t_{n}\right)\right\} \\
y\left(t_{n}\right) \leq f\left(t_{n}\right)+g\left(t_{n}\right) y\left(t_{n}\right)<y\left(t_{n}\right), \\
n \geq n_{1}
\end{gathered}
$$

And that is contradiction. Hence $y(t)$ is bounded, let $\lim \sup _{t \rightarrow \infty} y(t)=M_{1}<\infty$.

From (4) we get

$y(t)<\max \{y(s): \tau(t) \leq s \leq \gamma(t)\}, t \geq t_{0}$

By taking superior limit to both sides of the above inequality it follows that $M_{1}<M_{1}$. And that is contradiction.

Remark 1: In the following lemma, suppose that: 
$W(t)$

$=x(t)-P(t) x(\tau(t))$

$-\int_{\alpha^{-1}(\delta(t))}^{t} R(u) x(\alpha(u)) d u$

$-\int_{t}^{\sigma^{-1}(\delta(t))} Q(u) x(\sigma(u)) d u$

Where $\delta(t)>t, \sigma(t)<t$ and $\alpha(t)>t$,

$t \in\left(t_{k}, t_{k+1}\right]$,

$0<t_{0}<t_{1}<\cdots<t_{k} \rightarrow \infty$ as $k \rightarrow \infty$.

Let $\alpha^{-1}(\delta(t)) \leq t$ and $\sigma^{-1}(\delta(t))>t$,

in addition to the following assumptions:

$\mathrm{H} 1:\left[R\left(\alpha^{-1}(\delta(t))\right)\left(\alpha^{-1}(\delta(t))\right)^{\prime}\right.$

$$
\begin{aligned}
& \left.-Q\left(\sigma^{-1}(\delta(t))\right)\left(\sigma^{-1}(\delta(t))\right)^{\prime}\right] \\
& \geq 0, t \geq t_{0}, t \in\left(t_{k}, t_{k+1}\right]
\end{aligned}
$$

H2: There exist positive real numbers $a_{k}$ and $b_{k}$ such that

$$
a_{k}-b_{k} \geq 1, k=1,2, \ldots \text { and }
$$

$\int P\left(t_{k}^{+}\right) \leq P\left(t_{k}\right)$ for $\tau\left(t_{k}\right) \neq t_{i}, i<k$,

$\left\{\begin{array}{c}P\left(t_{k}^{+}\right) \leq \frac{1}{a_{k}-b_{k}} P\left(t_{k}\right) \text { for } \tau\left(t_{k}\right)=t_{i}, i<k,\end{array}\right.$ $a_{k}=a_{i}, b_{k}=b_{i}$ when

$$
\tau\left(t_{k}\right)=t_{i}, i<k .
$$

Lemma 4: Let $W(t)$ be defined as in (6),

H1-H2 hold, and

$$
\begin{aligned}
& \liminf _{\mathrm{t} \rightarrow \infty} \int_{t}^{\delta(t)}\left[R\left(\alpha^{-1}(\delta(\mathrm{u}))\right)\left(\alpha^{-1}(\delta(\mathrm{u}))\right)^{\prime}\right. \\
& \left.-Q\left(\sigma^{-1}(\delta(\mathrm{u}))\right)\left(\sigma^{-1}(\delta(\mathrm{u}))\right)^{\prime}\right] d u>\frac{1}{e^{\prime}} \\
& t \in\left(t_{k}, t_{k+1}\right] . \quad(7)
\end{aligned}
$$

Let $x(t)$ be an eventually positive solution of Eq.(1). Then $W(t)$ is eventually negative and nondecreasing function.

Proof. Let $x(t)$ be an eventually positive solution of Eq.(1), that is $x(t)>0$,

$x(\tau(t))>0, \quad x(\sigma(t))>0$ and $x(\alpha(t))>0, t \geq$ $t_{0}$,

Differentiate Eq.(6) for every interval $\left(t_{k}, t_{k+1}\right]$ where $\mathrm{k}=1,2, \ldots$ and use Eq.(1), then:

$W^{\prime}(t)$

$=[x(t)-P(t) x(\tau(t))]^{\prime}-R(t) x(\alpha(t))$

$+R\left(\alpha^{-1}(\delta(t))\right) x(\delta(t))\left(\alpha^{-1}(\delta(t))\right)^{\prime}$

$-Q\left(\sigma^{-1}(\delta(t))\right) x(\delta(t))\left(\sigma^{-1}(\delta(t))\right)^{\prime}$

$+Q(t) x(\sigma(t))$

$=-Q(t) x(\sigma(t))+R(t) x(\alpha(t))-R(t) x(\alpha(t))$

$+R\left(\alpha^{-1}(\delta(t))\right) x(\delta(t))\left(\alpha^{-1}(\delta(t))\right)^{\prime}$

$-Q\left(\sigma^{-1}(\delta(t))\right) x(\delta(t))\left(\sigma^{-1}(\delta(t))\right)^{\prime}$

$+Q(t) x(\sigma(t))$

$=\left[R\left(\alpha^{-1}(\delta(t))\right)\left(\alpha^{-1}(\delta(t))\right)^{\prime}\right.$

$\left.-Q\left(\sigma^{-1}(\delta(t))\right)\left(\sigma^{-1}(\delta(t))\right)^{\prime}\right] x(\delta(t))$

$\geq 0$
Hence $W(t)$ is nondecreasing function on

$t_{k}<t \leq t_{k+1}, k=1,2,3, \ldots$.

To prove that $W\left(t_{k}^{+}\right) \geq W\left(t_{k}\right)$ for $\mathrm{k}=1,2, \ldots$ In view of $a_{k}-b_{k} \geq 1$ and condition $\mathrm{H} 2$ when $\tau\left(t_{k}\right)=t_{i}, i<k$, then:

$W\left(t_{k}^{+}\right)=\left(a_{k}-b_{k}\right) x\left(t_{k}\right)-$

$P\left(t_{k}^{+}\right)\left(a_{k}-b_{k}\right) x\left(\tau\left(t_{k}\right)\right)$

$$
\begin{aligned}
& -\int_{\alpha^{-1}\left(\delta\left(t_{k}\right)\right)}^{t_{k}} R(u) x(\alpha(u)) d u \\
& -\int_{t_{k}}^{\sigma^{-1}\left(\delta\left(t_{k}\right)\right)} Q(u) x(\sigma(u)) d u \\
\geq x\left(t_{k}\right)-P\left(t_{k}\right) & x\left(\tau\left(t_{k}\right)\right) \\
& -\int_{\alpha^{-1}\left(\delta\left(t_{k}\right)\right)}^{t_{k}} R(u) x(\alpha(u)) d u \\
& -\int_{t_{k}}^{\sigma^{-1}\left(\delta\left(t_{k}\right)\right)} Q(u) x(\sigma(u)) d u \\
& =W\left(t_{k}\right) .
\end{aligned}
$$

When $\tau\left(t_{k}\right) \neq t_{i}, i<k$ then from $\mathrm{H} 2$

$$
\begin{gathered}
W\left(t_{k}^{+}\right)=\left(a_{k}-b_{k}\right) x\left(t_{k}\right)-P\left(t_{k}^{+}\right) x\left(\tau\left(t_{k}\right)\right) \\
-\int_{\alpha^{-1}\left(\delta\left(t_{k}\right)\right)}^{t_{k}} R(u) x(\alpha(u)) d u \\
-\int_{t_{k}}^{\sigma^{-1}\left(\delta\left(t_{k}\right)\right)} Q(u) x(\sigma(u)) d u \\
\geq\left(a_{k}-b_{k}\right) x\left(t_{k}\right)-P\left(t_{k}\right) x\left(\tau\left(t_{k}\right)\right) \\
-\int_{\alpha^{-1}\left(\delta\left(t_{k}\right)\right)}^{t_{k}} R(u) x(\alpha(u)) d u \\
-\int_{t_{k}}^{\sigma^{-1}\left(\delta\left(t_{k}\right)\right)} Q(u) x(\sigma(u)) d u \\
\geq W\left(t_{k}\right) \quad(9)
\end{gathered}
$$

$W(t)$ is nondecreasing on $\left[t_{0}, \infty\right)$. Hence $-\infty<$ $\lim _{t \rightarrow \infty} W(t)=L \leq \infty$. Where

$|L|=\sup \left\{W\left(t_{k}^{+}\right), \lim _{k \rightarrow \infty} W\left(t_{k}\right)\right\}, t \in\left[t_{l}, \infty\right)$

We claim that $W(t) \leq 0$ for $t \in\left(t_{k}, t_{k+1}\right]$, $k=l, l+1, \ldots$. Otherwise, there exists

$t^{*} \in\left(t_{k}, t_{k+1}\right]$ such that $W\left(t^{*}\right)>0$. So $W(t) \geq$ $W\left(t^{*}\right)>0, t \geq t^{*}, W(t) \leq x(t)$ for

$t \in\left(t_{k}, t_{k+1}\right], W(\delta(t)) \leq x(\delta(t))$ and $W^{\prime}(t)$

$$
\begin{aligned}
& =\left[R\left(\alpha^{-1}(\delta(t))\right)\left(\alpha^{-1}(\delta(t))\right)^{\prime}\right. \\
& \left.-Q\left(\sigma^{-1}(\delta(t))\right)\left(\sigma^{-1}(\delta(t))\right)^{\prime}\right] x(\delta(t)) \\
& \geq\left[R\left(\alpha^{-1}(\delta(t))\right)\left(\alpha^{-1}(\delta(t))\right)^{\prime}\right. \\
& \left.-Q\left(\sigma^{-1}(\delta(t))\right)\left(\sigma^{-1}(\delta(t))\right)^{\prime}\right] W(\delta(t)) \\
& \quad W^{\prime}(t)-\left[R\left(\alpha^{-1}(\delta(t))\right)\left(\alpha^{-1}(\delta(t))\right)^{\prime}\right. \\
& \left.-Q\left(\sigma^{-1}(\delta(t))\right)\left(\sigma^{-1}(\delta(t))\right)^{\prime}\right] W(\delta(t)) \geq 0
\end{aligned}
$$

In view of condition (7) and Lemma 1 , the last inequality cannot has eventually positive solution, 
which is a contradiction. Hence $W(t) \leq 0$ for $t \in\left(t_{k}, t_{k+1}\right], k=l, l+1, \ldots$

It remains to prove that $W\left(t_{k}\right)<0$ for

$k=1,2, \ldots$. If it is not true, then there exists $t_{m} \in\left(t_{k}, t_{k+1}\right], \quad$ such that $W\left(t_{m}\right)=0$, by integrating (8) on $\left(t_{m}, t_{m+1}\right]$, we have:

$$
\begin{gathered}
W\left(t_{m+1}\right)=W\left(t_{m}^{+}\right) \\
+\int_{t_{m}}^{t_{m+1}}\left[Q\left(\sigma^{-1}(\delta(t))\right)\left(\sigma^{-1}(\delta(t))\right)^{\prime}\right. \\
\left.-R\left(\alpha^{-1}(\delta(t))\right)\left(\alpha^{-1}(\delta(t))\right)^{\prime}\right] x(\delta(t)) d t \\
\geq W\left(t_{m}^{+}\right) \geq W\left(t_{m}\right)=0
\end{gathered}
$$

This contradiction shows that

$W\left(t_{k}\right)<0$ for $k=1,2 \ldots$ therefore from (8)

$W(t) \leq W\left(t_{k+1}\right)<0$, where $t_{k}<t \leq t_{k+1}, k=$ $1,2, \ldots$

Thus $W(t)<0$ for $t \geq t_{0}$.

Remark 2: In the following lemma, suppose that:

Let $\alpha^{-1}(\delta(t)) \leq t$ and $t \leq \sigma^{-1}(\delta(t))$, and $\mathrm{H} 4:\left[Q\left(\sigma^{-1}(\delta(t))\right)\left(\sigma^{-1}(\delta(t))\right)^{\prime}\right.$

$$
\begin{aligned}
& \left.-R\left(\alpha^{-1}(\delta(t))\right)\left(\alpha^{-1}(\delta(t))\right)^{\prime}\right] \\
& \geq 0, t \geq t_{0}, \quad t \in\left(t_{k}, t_{k+1}\right]
\end{aligned}
$$

H5: There exists nonnegative real numbers $a_{k}$ and $b_{k}, 0<a_{k}-b_{k} \leq 1$,

$k=1,2, \ldots$ such that

$\int P\left(t_{k}^{+}\right) \geq P\left(t_{k}\right)$ for $\tau\left(t_{k}\right) \neq t_{i}, i<k$,

$P\left(t_{k}^{+}\right) \geq \frac{1}{a_{k}-b_{k}} P\left(t_{k}\right)$ for $\tau\left(t_{k}\right)=t_{i}, i<k$, where

$a_{k}=a_{i}, b_{k}=b_{i}$ when $\tau\left(t_{k}\right)=t_{i}$,

$$
i<k
$$

Lemma 5. Assume that H4-H5 hold. Let $x(t)$ be an eventually positive solution of Eq.(1) and

$$
\begin{gathered}
\liminf _{\mathrm{t} \rightarrow \infty} \int_{\delta(t)}^{t}\left[Q\left(\sigma^{-1}(\delta(\mathrm{u}))\right)\left(\sigma^{-1}(\delta(\mathrm{u}))\right)^{\prime}\right. \\
\left.-R\left(\alpha^{-1}(\delta(\mathrm{u}))\right)\left(\alpha^{-1}(\delta(\mathrm{u}))\right)^{\prime}\right] d u>\frac{1}{e^{\prime}} \\
t \in\left(t_{k}, t_{k+1}\right] .
\end{gathered}
$$

Where $\quad \delta(t)<t, t \in\left(t_{k}, t_{k+1}\right], 0<t_{0}<t_{1}<$ $\cdots<t_{k} \rightarrow \infty$ as $k \rightarrow \infty$. Let $W(t)$ be defined as in (6). Then $W(t)$ is eventually negative and nonincreasing function.

Proof. Let $x(t)$ be an eventually positive solution of equation that is $x(t)>0, x(\tau(t))>$ $0, x(\sigma(t))>0$ and $x(\alpha(t))>0, t \geq t_{0}$,

Differentiate (6) for every interval $\left(t_{k}, t_{k+1}\right]$ where $k=1,2, \ldots$ and use Eq.(1),then:

$W^{\prime}(t)$

$=[x(t)-P(t) x(\tau(t))]^{\prime}-R(t) x(\alpha(t))$

$+R\left(\alpha^{-1}(\delta(t))\right) x(\delta(t))\left(\alpha^{-1}(\delta(t))\right)^{\prime}$

$-Q\left(\sigma^{-1}(\delta(t))\right) x(\delta(t))\left(\sigma^{-1}(\delta(t))\right)^{\prime}$

$+Q(t) x(\sigma(t))$

$$
\begin{gathered}
=-Q(t) x(\sigma(t))+R(t) x(\alpha(t))-R(t) x(\alpha(t)) \\
+R\left(\alpha^{-1}(\delta(t))\right) x(\delta(t))\left(\alpha^{-1}(\delta(t))\right)^{\prime} \\
-Q\left(\sigma^{-1}(\delta(t))\right) x(\delta(t))\left(\sigma^{-1}(\delta(t))\right)^{\prime} \\
+Q(t) x(\sigma(t)) \\
=-\left[Q\left(\sigma^{-1}(\delta(t))\right)\left(\sigma^{-1}(\delta(t))\right)^{\prime}\right) \\
\left.-R\left(\alpha^{-1}(\delta(t))\right)\left(\alpha^{-1}(\delta(t))\right)^{\prime}\right] x(\delta(t))
\end{gathered}
$$

Then: $W^{\prime}(t) \leq 0$.

Hence $W(t)$ is nonincreasing function on

$$
t_{k}<t \leq t_{k+1} \text { for } k \geq 0
$$

To prove that $W\left(t_{k}^{+}\right) \leq W\left(t_{k}\right)$ for $k=1,2, \ldots$ In view of $0<a_{k}-b_{k} \leq 1$ and from (6) with regard to $\mathrm{H} 5$ when $\tau\left(t_{k}\right)=t_{i}, i<k$, then:

$$
\begin{array}{r}
W\left(t_{k}^{+}\right)=\left(a_{k}-b_{k}\right) x\left(t_{k}\right) \\
-P\left(t_{k}^{+}\right)\left(a_{k}-b_{k}\right) x\left(\tau\left(t_{k}\right)\right) \\
-\int_{\alpha^{-1}\left(\delta\left(t_{k}\right)\right)}^{t_{k}} R(u) x(\alpha(u)) d u \\
-\int_{t_{k}}^{\sigma^{-1}\left(\delta\left(t_{k}\right)\right)} Q(u) x(\sigma(u)) d u \\
\leq x\left(t_{k}\right)-P\left(t_{k}\right) x\left(\tau\left(t_{k}\right)\right) \\
-\int_{\alpha^{-1}\left(\delta\left(t_{k}\right)\right)}^{t_{k}} R(u) x(\alpha(u)) d u \\
-\int_{t_{k}}^{\sigma^{-1}\left(\delta\left(t_{k}\right)\right)} Q(u) x(\sigma(u)) d u
\end{array}
$$$$
=W\left(t_{k}\right)
$$

When $\tau\left(t_{k}\right) \neq t_{i}, i<k$ then from (6) with regard to the condition $\mathrm{H} 5$ :

$$
\begin{gathered}
W\left(t_{k}^{+}\right)=\left(a_{k}-b_{k}\right) x\left(t_{k}\right)-P\left(t_{k}^{+}\right) x\left(\tau\left(t_{k}\right)\right) \\
-\int_{\alpha^{-1}\left(\delta\left(t_{k}\right)\right)}^{t_{k}} R(u) x(\alpha(u)) d u \\
-\int_{t_{k}}^{\sigma^{-1}\left(\delta\left(t_{k}\right)\right)} Q(u) x(\sigma(u)) d u \\
\leq\left(a_{k}-b_{k}\right) x\left(t_{k}\right)-P\left(t_{k}\right) x\left(\tau\left(t_{k}\right)\right) \\
-\int_{\alpha^{-1}\left(\delta\left(t_{k}\right)\right)}^{t_{k}} R(u) x(\alpha(u)) d u \\
-\int_{t_{k}}^{\sigma^{-1}\left(\delta\left(t_{k}\right)\right)} Q(u) x(\sigma(u)) d u
\end{gathered}
$$

$\leq W\left(t_{k}\right)$

$W(t)$ is nonincreasing on $\left[t_{0}, \infty\right)$,

$-\infty \leq \lim _{t \rightarrow \infty} W(t)=L<\infty$. Where

$|L|=\sup \left\{W\left(t_{k}^{+}\right), \lim _{k \rightarrow \infty} W\left(t_{k}\right)\right\}, t \in\left[t_{l}, \infty\right)$

Suppose that $W(t) \leq 0$ for $t \in\left(t_{k}, t_{k+1}\right]$,

$k=l, l+1, \ldots$. Otherwise, there exists

$t^{*} \in\left(t_{k}, t_{k+1}\right]$ such that

$W\left(t^{*}\right)>0$. So $W(t) \geq W\left(t^{*}\right)>0$,

$t_{k}<t \leq t^{*}$

$W(t) \leq x(t)$ for $t \in\left(t_{k}, t_{k+1}\right]$

$W(\delta(t)) \leq x(\delta(t))$ and 


$$
\begin{gathered}
W^{\prime}(t) \\
=-\left[Q\left(\sigma^{-1}(\delta(t))\right)\left(\sigma^{-1}(\delta(t))\right)^{\prime}\right. \\
\left.-R\left(\alpha^{-1}(\delta(t))\right)\left(\alpha^{-1}(\delta(t))\right)^{\prime}\right] x(\delta(t)) \\
W^{\prime}(t) \\
\leq-\left[Q\left(\sigma^{-1}(\delta(t))\right)\left(\sigma^{-1}(\delta(t))\right)^{\prime}\right. \\
\left.-R\left(\alpha^{-1}(\delta(t))\right)\left(\alpha^{-1}(\delta(t))\right)^{\prime}\right] W(\delta(t)) \\
W^{\prime}(t)+\left[Q\left(\sigma^{-1}(\delta(t))\right)\left(\sigma^{-1}(\delta(t))\right)^{\prime}\right. \\
\left.-R\left(\alpha^{-1}(\delta(t))\right)\left(\alpha^{-1}(\delta(t))\right)^{\prime}\right] W(\delta(t)) \leq 0 .
\end{gathered}
$$

With regard to (10) and Lemma 2, the last inequality cannot has eventually positive solution, which is a contradiction. Hence $W(t) \leq 0$ for $t \in\left(t_{k}, t_{k+1}\right], k=l, l+1, \ldots$.

It remains to prove that $W\left(t_{k}\right)<0$ for $k=1,2, \ldots$. If it is not true, then there exists $t_{m} \in\left(t_{k}, t_{k+1}\right], \quad$ such that $W\left(t_{m}\right)=0$, and from (11), we have:

$$
\begin{aligned}
& W\left(t_{m+1}\right) \\
& =W\left(t_{m}^{+}\right) \\
& -\int_{t_{m}}^{t_{m+1}}\left[Q\left(\sigma^{-1}(\delta(t))\right)\left(\sigma^{-1}(\delta(t))\right)^{\prime}\right. \\
& \left.-R\left(\alpha^{-1}(\delta(t))\right)\left(\alpha^{-1}(\delta(t))\right)^{\prime}\right] x(\delta(t)) d t \\
& \quad \leq W\left(t_{m}^{+}\right) \leq W\left(t_{m}\right)=0
\end{aligned}
$$

This contradiction shows that $W\left(t_{k}\right)<0$ for $k=1,2 \ldots$ therefore from (11) it follows that $W(t) \leq W\left(t_{k}\right)<0$, where

$t_{k}<t \leq t_{k+1}, k=1,2, \ldots$.

Thus $W(t)<0$ for $t \geq t_{0}$.

\section{Main results}

Remark 3: In the following theorems, if $y(t)=$ $x(t)$ almost everywhere, where

$y(t) \in C\left(\left[t_{0}, \infty\right) ; R\right)$ and $x(t) \in P C\left(\rho\left(t_{0}\right), R\right)$.

The next results provide sufficient conditions for the oscillation of all solutions of Eq.(1):

Theorem 1. Let $W(t)$ be defined as in (6) and the assumptions $\mathrm{H} 1-\mathrm{H} 2$ and (7) hold, in addition to the condition

$$
\begin{aligned}
& P(t)+\int_{\alpha^{-1}(\delta(t))}^{t} R(u) d u+\int_{t}^{\sigma^{-1}(\delta(t))} Q(u) d u \\
& \leq 1, t \geq t_{0} .
\end{aligned}
$$

Where $t_{k}<t<\delta(t) \leq t_{k+1}$,

$$
\begin{aligned}
t_{k}<t \leq \tau^{-1}(\delta(u)) \leq t_{k+1}, k=l, l+ \\
1, \ldots, t_{k}<\alpha^{-1}(\delta(t))<t \leq t_{k+1}, \\
t_{k}<t<\sigma^{-1}(\delta(t)) \leq t_{k+1} .
\end{aligned}
$$

Then every bounded solution of Eq.(1) oscillates.

Proof. Assume that $x(t)$ be bounded eventually positive solution of Eq.(1). By lemma 4 it follows that $W(t)$ is eventually negative and nondecreasing, from (6) we get:

$$
\begin{aligned}
& x(t)=W(t)+P(t) x(\tau(t)) \\
& +\int_{\alpha^{-1}(\delta(t))}^{t} R(u) x(\alpha(u)) d u \\
& +\int_{t}^{\sigma^{-1}(\delta(t))} Q(u) x(\sigma(u)) d u \\
& \leq W(t) \\
& +\left(P(t)+\int_{\alpha^{-1}(\delta(t))}^{t} R(u) d u\right. \\
& \left.+\int_{t}^{\sigma^{-1}(\delta(t))} Q(u) d u\right) \max \{x(s): \rho(t) \leq s \\
& \left.\leq \sigma^{-1}(\delta(t))\right\} \text {, } \\
& \rho(t)=\min \left\{\tau(t), \alpha^{-1}(\delta(t))\right\} \\
& \leq W(t)+\max \{x(s): \rho(t) \leq s \\
& \left.\leq \sigma^{-1}(\delta(t))\right\}
\end{aligned}
$$

By lemma 3, we get $x(t)$ is eventually negative, a contradiction.

Theorem 2. Let $W(t)$ be defined as in (6), and let H4 - H5, (10) and (13) hold, Then every bounded solution of Eq.(1) oscillates.

Proof. Assume that $x(t)$ be eventually positive solution of Eq.(1.1). From lemma 5, it follows that $W(t)$ is eventually negative and nonincreasing. From (6) for $t \geq t_{1} \geq t_{0}$ we get:

$$
\begin{aligned}
x(t)=W(t)+ & P(t) x(\tau(t)) \\
& +\int_{\alpha^{-1}(\delta(t))}^{t} R(u) x(\alpha(u)) d u \\
& +\int_{t}^{\sigma^{-1}(\delta(t))} Q(u) x(\sigma(u)) d u \\
x(t) \leq W\left(t_{1}\right)+ & P(t) x(\tau(t)) \\
& +\int_{\alpha^{-1}(\delta(t))}^{t} R(u) x(\alpha(u)) d u \\
& +\int_{t}^{\sigma^{-1}(\delta(t))} Q(u) x(\sigma(u)) d u
\end{aligned}
$$$$
\leq W\left(t_{1}\right)
$$$$
+\left(P(t)+\int_{\alpha^{-1}(\delta(t))}^{t} R(u) d u\right.
$$$$
\left.+\int_{t}^{\sigma^{-1}(\delta(t))} Q(u) d u\right) \max \{x(s): \rho(t) \leq s
$$$$
\left.\leq \sigma^{-1}(\delta(t))\right\}
$$

$$
\leq W\left(t_{1}\right)+\max \left\{x(s): \rho(t) \leq s \leq \sigma^{-1}(\delta(t))\right\},
$$

where $\rho(t)=\min \left\{\tau(t), \alpha^{-1}(\delta(t))\right\}$.

By lemma 3 , it follows that $x(t)$ is eventually negative, leads to a contradiction.

Example 1: Consider the impulsive neutral differential equation 


$$
\left.\begin{array}{c}
{\left[x(t)-e^{-\pi}\left(1-e^{-t}\right) x(t-2 \pi)\right]^{\prime}+} \\
e^{-t} x(t-2 \pi)- \\
\left(1-e^{-\pi}+e^{-\pi} e^{-t}\right) x\left(t+\frac{\pi}{2}\right)=0, \\
t \neq t_{k}, k=1,2, \ldots \\
x\left(t_{k}^{+}\right)=\frac{k+1}{k} x\left(t_{k}\right), \quad t=t_{k} \\
k=1,2, \ldots
\end{array}\right\}
$$

Let

$$
\begin{aligned}
& x(t)=\left\{\begin{array}{ll}
\sin t, & t \neq t_{k} \\
1+\frac{1}{k}, & t=t_{k},
\end{array} \quad P(t)=\right. \\
& \begin{cases}e^{-\pi}\left(1-e^{-t}\right), & t \neq t_{k} \\
k, & t=t_{k}\end{cases}
\end{aligned}
$$

Where $a_{k}=\frac{k+2}{k}$ and $b_{k}=\frac{1}{k}$.

We can see that where $\delta(t)=t+\frac{\pi}{4}$.

$$
a_{k}-b_{k}=\frac{k+2}{k}-\frac{1}{k}=\frac{k+1}{k}>1,
$$

$$
\begin{gathered}
\mathrm{H} 1:\left[R\left(\alpha^{-1}(\delta(t))\right)\left(\alpha^{-1}(\delta(t))\right)^{\prime}\right. \\
\left.-Q\left(\sigma^{-1}(\delta(t))\right)\left(\sigma^{-1}(\delta(t))\right)^{\prime}\right] \\
\begin{aligned}
=R\left(t-\frac{\pi}{4}\right)-Q\left(t+\frac{9 \pi}{4}\right) \\
=\left(1-e^{-\pi}+e^{-\pi} e^{-t+\frac{\pi}{4}}\right)-e^{-\pi} e^{-t-\frac{9 \pi}{4}} \\
=0.956786+0.094744 e^{-t}>0, \\
t \in\left(t_{k}, t_{k+1}\right] . \\
P\left(t_{k}^{+}\right)=P\left(k^{+}\right)=\lim _{t \rightarrow k^{+}} P(t) \\
=\lim _{t \rightarrow k^{+}} e^{-\pi}\left(1-e^{-t}\right)=e^{-\pi}\left(1-e^{-k}\right) \\
\leq 0.043213 \\
<\frac{k^{2}}{k+1}=\frac{1}{a_{k}-b_{k}} P\left(t_{k}\right)
\end{aligned}
\end{gathered}
$$

for $k=1,2, \ldots$

$$
\begin{array}{r}
P\left(t_{k}^{+}\right)=0.043213<\frac{k+1}{k} k=k+1 \\
=\left(a_{k}-b_{k}\right) P\left(t_{k}\right) \text { for } k=1,2, \ldots
\end{array}
$$

So, $\mathrm{H} 2$ holds.

$$
\begin{gathered}
\liminf _{\mathrm{t} \rightarrow \infty} \int_{t}^{\delta(t)}\left[R\left(\alpha^{-1}(\delta(\mathrm{u}))\right)\left(\alpha^{-1}(\delta(\mathrm{u}))\right)^{\prime}\right. \\
\left.-Q\left(\sigma^{-1}(\delta(\mathrm{u}))\right)\left(\sigma^{-1}(\delta(\mathrm{u}))\right)^{\prime}\right] d u \\
=\liminf _{\mathrm{t} \rightarrow \infty} \int_{t}^{t+\frac{\pi}{4}}\left[R\left(u-\frac{\pi}{4}\right)\right. \\
\left.-Q\left(u+\frac{9 \pi}{4}\right)\right] d u \\
=\liminf _{\mathrm{t} \rightarrow \infty} \int_{t}^{t+\frac{\pi}{4}}\left[0.956786+0.094744 e^{-u}\right] d u \\
=0.751457>\frac{1}{e}
\end{gathered}
$$

$$
\begin{array}{r}
{\left[P(t)+\int_{\alpha^{-1}(\delta(t))}^{t} R(u) d u+\int_{t}^{\sigma^{-1}(\delta(t))} Q(u) d u\right]} \\
=\left[e^{-\pi}\left(1-e^{-t}\right)\right. \\
\quad+\int_{t-\frac{\pi}{4}}^{t}\left(1-e^{-\pi}+e^{-\pi} e^{-u}\right) d u \\
\left.+\int_{t}^{t+\frac{9 \pi}{4}} e^{-\pi} e^{-u} d u\right]
\end{array}
$$

$=0.845385<1$. Hence all conditions of theorem 1 are fulfilled, then according to theorem 1 all solutions of equation (14) oscillate. It is obvious that

$x(t)=\left\{\begin{array}{ll}\sin t, & t \neq t_{k} \\ 1+\frac{1}{k}, & t=t_{k}\end{array} \quad\right.$ is such an oscillatory solution.

Example 2: Consider the impulsive neutral differential equation

$$
\begin{gathered}
{\left[x(t)-e^{-2 \pi}\left(1-e^{-t}\right) x(t-2 \pi)\right]^{\prime}+} \\
\left(1-e^{-2 \pi}+e^{-2 \pi} e^{-t}\right) x\left(t-\frac{\pi}{2}\right) \\
-e^{-2 \pi} e^{-t} x(t+\pi)=0 \\
t \neq t_{k}, k=1,2, \ldots \\
x\left(t_{k}^{+}\right)=\frac{k+1}{k+2} x\left(t_{k}\right), \quad t=t_{k} \\
k=1,2, \ldots
\end{gathered}
$$

Let

$$
\begin{aligned}
& x(t)=\left\{\begin{array}{cc}
\cos t, & t \neq t_{k} \\
1+\frac{1}{5 k}, & t=t_{k}
\end{array}, \quad \mathrm{P}(\mathrm{t})=\right. \\
& \left\{\begin{array}{cc}
e^{-2 \pi}\left(1-e^{-t}\right), & t \neq t_{k} \\
\frac{1}{1500 k}, \quad t=t_{k}
\end{array}\right.
\end{aligned}
$$

Where $a_{k}=\frac{2 k+2}{k+2}$ and $b_{k}=\frac{k+1}{k+2}$.

We can see that $a_{k}-b_{k}=\frac{2 k+2}{k+2}-\frac{k+1}{k+2}=\frac{k+1}{k+2}<1$

Let $t_{k}=k, P\left(t_{k}^{+}\right)=P\left(k^{+}\right)$

$$
\begin{gathered}
=\lim _{t \rightarrow k^{+}} P(t)=\lim _{t \rightarrow k^{+}} e^{-2 \pi}\left(1-e^{-t}\right) \\
=e^{-2 \pi}\left(1-e^{-k}\right)
\end{gathered}
$$

So $0 \leq P\left(t_{k}^{+}\right) \leq 0.001867$,

if $k=1$ then $P\left(t_{1}^{+}\right)=0.00118$

$$
\begin{gathered}
\left(a_{k}-b_{k}\right) P\left(t_{k}\right)=\frac{k+1}{k+2} \frac{1}{1500 k} \\
=\frac{k+1}{1500 k(k+2)}
\end{gathered}
$$

If $k=1$ then $\quad\left(a_{1}-b_{1}\right) P\left(t_{1}\right)=0.000444$, and

$$
\begin{aligned}
& \frac{1}{\left(a_{k}-b_{k}\right)} P\left(t_{k}\right)=\frac{k+2}{k+1} \frac{1}{1500 k}=\frac{k+2}{1500 k(k+1)} \\
& \text { if } k=1 \text {, then } \frac{1}{\left(a_{1}-b_{1}\right)} P\left(t_{1}\right)=0.001 \text {, so H5 holds. } \\
& \text { Where } \delta(t)=t-\frac{\pi}{4} \text {. } \\
& \text { H4: }\left[R\left(\alpha^{-1}(\delta(t))\right)\left(\alpha^{-1}(\delta(t))\right)^{\prime}-\right. \\
& \left.Q\left(\sigma^{-1}(\delta(t))\right)\left(\sigma^{-1}(\delta(t))\right)^{\prime}\right]=R\left(t-\frac{5 \pi}{4}\right)- \\
& Q\left(t+\frac{\pi}{4}\right)
\end{aligned}
$$




$$
\begin{aligned}
& =e^{-2 \pi} e^{-t=\frac{5 \pi}{4}}-\left(1-e^{-2 \pi}\right. \\
& \left.\quad+e^{-2 \pi} e^{-t-\frac{\pi}{4}}\right) \\
& =-0.903376-0.00851 e^{-t}<0
\end{aligned}
$$

$t \in\left(t_{k}, t_{k+1}\right]$.

H5: $P\left(t_{k}^{+}\right)=\frac{1}{2} \leq \frac{k^{2}}{k+1} \leq \frac{1}{a_{k}-b_{k}} P\left(t_{k}\right), t \neq t_{k} \quad$ for $k=1,2, \ldots$

$$
\begin{gathered}
P\left(t_{k}^{+}\right)=\frac{1}{2}<\frac{k+1}{k} k=k+1=\left(a_{k}-\right. \\
\left.b_{k}\right) P\left(t_{k}\right), t=t_{k} \text { for } k=1,2, \ldots \\
\begin{array}{c}
\liminf _{\mathrm{t} \rightarrow \infty} \int_{\delta(t)}^{t}\left[Q\left(\sigma^{-1}(\delta(\mathrm{u}))\right)\left(\sigma^{-1}(\delta(\mathrm{u}))\right)^{\prime}\right. \\
\left.\quad-R\left(\alpha^{-1}(\delta(\mathrm{u}))\right)\left(\alpha^{-1}(\delta(\mathrm{u}))\right)^{\prime}\right] d u \\
=\liminf _{\mathrm{t} \rightarrow \infty} \int_{t-\frac{\pi}{4}}^{t}\left[Q\left(u+\frac{\pi}{4}\right)-R\left(u-\frac{5 \pi}{4}\right)\right] d u \\
=\liminf _{\mathrm{t} \rightarrow \infty} \int_{t-\frac{\pi}{4}}^{t}\left[0.903376+0.00851 e^{-u}\right] d u \\
=0.709509>\frac{1}{e} \\
{\left[P(t)+\int_{\alpha^{-1}(\delta(t))}^{t} R(u) d u+\int_{t}^{\sigma^{-1}(\delta(t))} Q(u) d u\right]} \\
=e^{-2 \pi}\left(1-e^{-t}\right) \\
\quad+\int_{t-\frac{5 \pi}{4}}^{t} e^{-2 \pi} e^{-u} d u \\
+\int_{t}^{t+\frac{\pi}{4}}\left(1-e^{-2 \pi}\right. \\
\left.\left.+e^{-2 \pi} e^{-u}\right) d u\right]
\end{array} \\
=0.879703<1
\end{gathered}
$$

Hence all conditions of theorem 2 are fulfilled, then according to theorem 2 all solutions of equation (15) oscillate. It is obvious that

$$
x(t)=\left\{\begin{array}{l}
\cos t, \quad t \neq t_{k} \\
1+\frac{1}{5 k}, \quad t=t_{k}
\end{array}\right. \text { is such oscillatory }
$$

solution with impulsive.

\section{Conclusion:}

In this paper, the impulsive neutral differential equations are studied. The impulses characteristics of the first order neutral differential equations have been clarified. Some necessary and sufficient conditions have been obtained to ensure that all bounded solutions of the first order neutral differential equations are oscillatory. The lemma 1.5.4 in (14) has been generalized, some new lemmas have been submitted to obtain the main results of the oscillation property. Illustrative examples of the obtained results have been provided.

\section{Authors' declaration:}

- Conflicts of Interest: None.

- Ethical Clearance: The project was approved by the local ethical committee in University of Baghdad.

\section{References:}

1. Li X, Bohner M, Wang C. Impulsive differential equations: periodic solutions and application. Automatica, Elsevier. 2018; 52: 173-178.

2. Agarwal R, Hristova S, O'Regan D.Global Mittleffter Synchronization for Neural Networks by Impulsive Caputo Fractional Differential Equations with Distributed Delays. Symmety. 2018; 10(473): 120.

3. Stamov G T, Stamova I M. Almost periodic solutions for impulsive neural networks with delay. Appl Math Model 2007; 1263-1270.

4. Hristova S. and Stamova I., On the Mittag- Leffler Stabibity of Impulsive Fractional Neural Networks with Finite Delays. IJPAM. 2016; 109(1): 105-117.

5. Feng Y., Yu J., Li C., Huang T. and Che H., Linear Impulsive Control System with Impulse Time Windows. J Vib Control. 2017; 23(1): 1-18.

6. Girel S. and Craust F., Existence and stability of periodic solutions of an impulsive differential equation and application to CD8 T-cell differential. J Math Biol 2018; 76( 7): 1756-1795.

7. Guerrini L. The Solow-Swan model with a bounded population growth rate. J MATH ECON 2006; 42: 14-21.

8. Mohmad H. A, Ketab S N. Asymptotic behavior criteria for solutions of nonlinear third order neutral differential equations, J. Math. Computer Sci. 2017; 2017:325-331.

9. Chen H, He Z, Li J. Multiplicity of solutions for impulsive differential equation on the half-line via variational methods. Chen el al. Boundary Value Problems. 2016; 2016(14): 1-15.

10. Abasiekwere U I, Isaac I O, Lipscey Z. Classification of non-oscillatory solutions neutral delay impulsive differential equations. Global Journals of Science Frontier Research (F).2018; (18): 49-64.

11. Abasiekwere A, Esuabana I M, Isaac I O, Lipcsey Z. Osillations of second order impulsive differential equations with advanced arguments. Glob. J. Sci. Front. Res., Math. Decision Sci. 2018; 18: 25-32.

12. Karakoc F, Unal A, Bereketoglu H. Oscillation of a nonlinear impulsive differential equation system with piecewise constant argument. Adv Differ Equ 2018 Dec;2018(1):1-1.

13. Agarwal R P, Grace S R, O'Regan D. Oscillation Theory for Difference and Functional Differential Equations. Kluwer Academic Publishers. 2000.

14. Györi I, Ladas G. Oscillation Theory of Delay Differential Equations with Applications. Clarendon Press Oxford. 1991. 
معيارية التذبذب لحلول المعادلات التفاضلية المحايدة ذات المعاملات الموجبة والسالبة وتأثير النبضات

عقيل فالح جدوع 2

حسين علي محمد 1

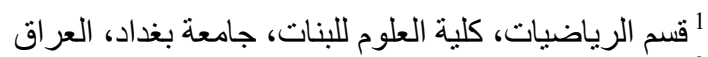

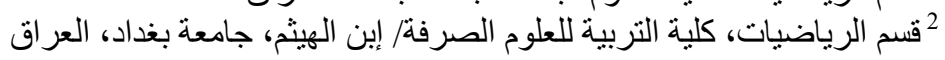

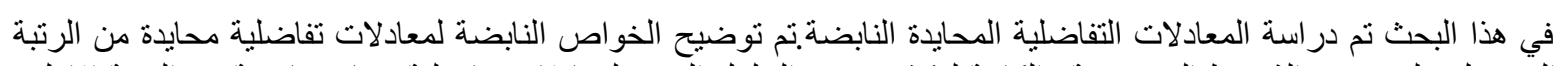
الخلاصة:

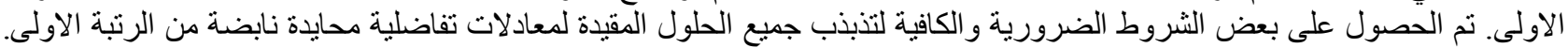

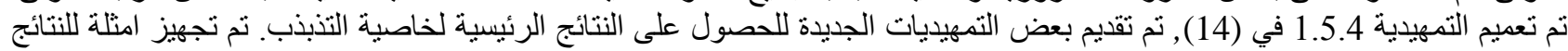
التي تم الحصول عليها.

الكلمات المفتاحية: معادلات تفاضلية محايدة نابضة، التذبذب، التباطؤات المتغيرة، المعاملات المتغيرة. 